\title{
BMJ Global Health Tracking changes in national BCG vaccination policies and practices using the BCG World Atlas
}

\author{
Samantha Lancione (D) , ${ }^{1}$ Jonathan Villa Alvarez, ${ }^{2}$ Hannah Alsdurf (D) , \\ Madhukar Pai (D) , ${ }^{3,4}$ Alice Anne Zwerling (D) ${ }^{1}$
}

To cite: Lancione S, Alvarez JV, Alsdurf $\mathrm{H}$, et al. Tracking changes in national BCG vaccination policies and practices using the BCG World Atlas. BMJ Global Health 2022;7:e007462. doi:10.1136/ bmjgh-2021-007462

Handling editor Seye Abimbola

- Additional supplemental material is published online only. To view, please visit the journal online (http://dx.doi.org/10. 1136/bmjgh-2021-007462)

Received 16 September 2021 Accepted 27 October 2021

\section{Check for updates}

(c) Author(s) (or their employer(s)) 2022. Re-use permitted under CC BY-NC. No commercial re-use. See rights and permissions. Published by BMJ.

${ }^{1}$ School of Epidemiology and Public Health, University of Ottawa, Ottawa, Ontario, Canada ${ }^{2}$ School of Medicine, University of Ottawa, Ottawa, Ontario, Canada

${ }^{3}$ McGill Internationa

Tuberculosis Centre, McGill University, Montreal, Québec, Canada

${ }^{4}$ Epidemiology \& Biostatistics McGill University, Montreal, Québec, Canada

Correspondence to Dr Alice Anne Zwerling; azwerlin@uottawa.ca

\section{ABSTRACT}

The $B C G$ vaccine is a widely given vaccine against tuberculosis (TB), yet studies on effectiveness have shown considerable heterogeneity; as a result, BCG vaccine policies vary greatly across the globe and change across geography, and with time and disease burden. The recently updated third BCG World Atlas (www.bcgatlas.org) is a publicly available online database with information on BCG practices across 194 countries. This helpful resource has been used for over 10 years to support clinicians, TB researchers and TB vaccine development worldwide. Here, we summarise main findings from the third BCG Atlas' most recent update which included additional data collected around BCG strain type, vaccine stockouts and associated changes. Longitudinal analysis enables evaluation of changes in TB incidence over time, a method becoming more common in legislation interventions. A large number of countries in the BCG Atlas (156/194 countries) maintain universal neonatal BCG vaccination, of which 51 are considered low TB burden countries. We demonstrate the majority of countries who changed their national policy moved to targeted vaccination for high-risk groups, were in Europe and also had significant decreases in TB incidence both before and after policy change. Globally, the most common BCG strain continues to be the Danish strain, despite its worldwide manufacturing interruption in 2015. Substantial variation and disproportionality exists in which regions were most affected by stockouts between 2009 and 2019. Tracking and understanding the reasoning behind changes to national BCG practices and their impact on TB burden is critical for decision makers as they contemplate how to include BCG vaccination in future immunisation guidelines in low and high TB burden countries.

\section{INTRODUCTION}

In 2019, an estimated 10 million people developed tuberculosis (TB) worldwide and 1.4 million died. ${ }^{1}$ There are several treatment options for TB, however, only one licensed vaccine, the BCG. Developed in 1921, the BCG is one of the world's oldest and most widely used vaccines, marking its 100th anniversary in 2021. ${ }^{1}$ WHO recommends BCG vaccination based on an individual country's
Summary box

- The BCG World Atlas (http://www.bcgatlas.org/) was created in 2011 as an online database containing details about global BCG vaccination policies and practices for over 194 countries and remains an important tool for members of the tuberculosis (TB) community.

- One-third of countries who continue to recommend universal vaccination in the BCG Atlas are considered to have low TB burden, raising important discussion around TB care infrastructure and surveillance.

- The majority of countries who modified their national BCG vaccine policy (either from a universal programme to targeting high-risk groups or modifying their definition of high-risk groups) were in Europe and had significant decreases in TB incidence before and after the policy change.

- Examining changes in BCG practices over the last decade and the reasoning behind them can help inform and guide policy-makers, researchers and vaccine developers to flag several areas for infrastructure strengthening that would benefit the global community.

TB epidemiology; countries with high TB burden should administer one dose to all neonates, while countries with low TB burden may target specific high-risk groups. ${ }^{1}$

Although effective in preventing severe forms of childhood TB, BCG effectiveness in adults is highly variable $(0 \%-80 \%)$ and continues to be debated. ${ }^{23}$ This uncertainty has led to wide regional variations and changes in BCG vaccine practices. ${ }^{2}$ For this reason, the BCG World Atlas (www.bcgatlas. org) was launched by our team in 2011 as an online database of current and past policies to be used by all members of the TB community (researchers, vaccine developers, clinicians, policy-makers, etc) worldwide. ${ }^{45}$ Data were collected from a range of sources including published literature, government documents and self-reported country questionnaires. 
Detailed methods related to data collection and creation of the database are published elsewhere. ${ }^{2}$

Countries may change national BCG practices for a variety of reasons but more countries are moving away from mass vaccination to target high-risk groups when TB incidence and burden have decreased significantly; this leads to lower risk of TB exposure and different risk/ benefit trade-offs for vaccination. ${ }^{6} 7$ The definition of high-risk groups also varies across countries and evolves within countries, changing with global recommendations, risk assessments and risk factors for TB infection. ${ }^{689}$

In 2015, one of the main BCG vaccine manufacturers, Statens Serum Institute-Denmark, reported a production failure causing a global supply shortage of 16.5 million doses. ${ }^{10}$ The effect of this major stockout and others over the last decade on BCG vaccine strain use is still not fully understood. ${ }^{10}$ Therefore, one of the aims of the recent update to the BCG Atlas was to collect more detailed data around BCG strain usage and stockouts. ${ }^{511}$

In spring of 2020, a data hackathon competition was developed as a collaboration between data scientists and our team to improve data gathering efforts and update the BCG Atlas. ${ }^{12}$ This collaboration was propelled by COVID-19; early studies were suggesting that the BCG vaccine may have non-specific immune boosting effects against COVID-19. ${ }^{13}{ }^{14}$ As global COVID-19 cases soared, more studies emerged examining the protective effect of BCG, and with them increasing anxiety of global BCG supply issues; concerns emerged that countries may hoard supplies and interrupt regular vaccination in high TB burden settings. ${ }^{15} 16$ These early ecological studies frequently used the BCG Atlas as a primary data source and highlighted knowledge gaps in BCG vaccination practices and the importance of having updated policy data. With the help of the hackathon, over 200 data points were extracted for 95 countries; new data was subsequently added to the online BCG Atlas and a third edition update was released in fall of $2020 .{ }^{11}$ The dataset now tracks over 45 key indicators of BCG vaccine policies and practices across 194 countries including, but not limited to, current BCG vaccination policy, booster doses, BCG strains, manufacturing interruptions and changes made to vaccination guidelines.

Taking advantage of the updated BCG Atlas, we can now explore the evolution of global BCG vaccination practices and policies, including the distribution of BCG strains and the frequency of BCG stockouts over the last decade. There are important unanswered questions about the impact of policy changes on national TB incidence rates, which can be addressed using resources such as the BCG World Atlas.

\section{BUILDING DATA ON BCG VACCINE POLICIES AND PRACTICES}

The BCG World Atlas (figure 1) is an online tool that contains information around BCG-related practices in a given country, enabling researchers and other members of the TB community to compare across countries. Countries are categorised based on their World Bank (WB) region and we used this classification to better understand and describe national and global changes related to BCG vaccine practices and policies.

As shown in figure 2, we compared current national BCG vaccine policy type, TB incidence rates in 2019 and WB region to inspect any patterns that may appear in the BCG Atlas. Data for yearly TB incidence rates (per 100000 population) were collected from the WHO publicly available monitoring system. These estimates are based on annual case notifications, assessments of TB notification data, national TB disease prevalence surveys and death registration systems. All forms of TB are included, including cases in people living with HIV.

Countries who changed their national BCG vaccine policy or practice between 2009 and 2019 were identified and categorised based on their type of policy change. TB
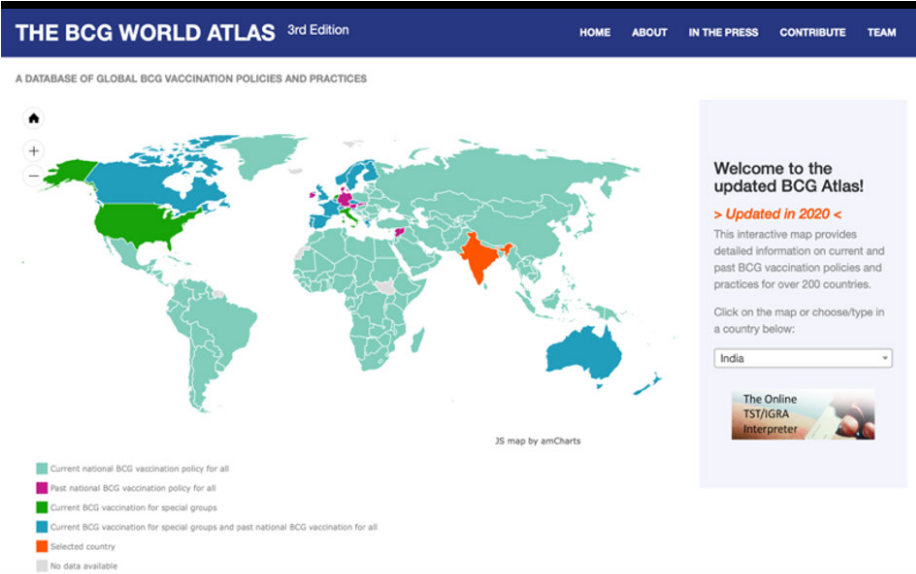

\section{India}

Last updated in 2020

Region

Income group (World Bank)

TB Incidence (per 100000 per year) ${ }^{\circ} \dagger$

TB Incidence (Count) ${ }^{*} \neq$

Current BCG vaccination?

BCG Recommendation Type

First appearance of BCG vaccine

(unofficial)

Which year was vaccination introduced?

Timing of 1st BCG?

Multiple BCG?

Multiple BCG in the past?

Year of $\mathrm{BCG}$ coverage estimate

Figure 1 Home page of the BCG World Atlas - third edition (http://www.bcgatlas.org/) and partial example of BCG policies and practices in India. TB, tuberculosis. *Data from WHO Statistical Information System for the year 2019. † Estimated number of new TB cases per 100000 population in 2019. ‡ Estimated number of new TB cases in the population in 2019. All forms of TB are included, including cases in people with HIV. 


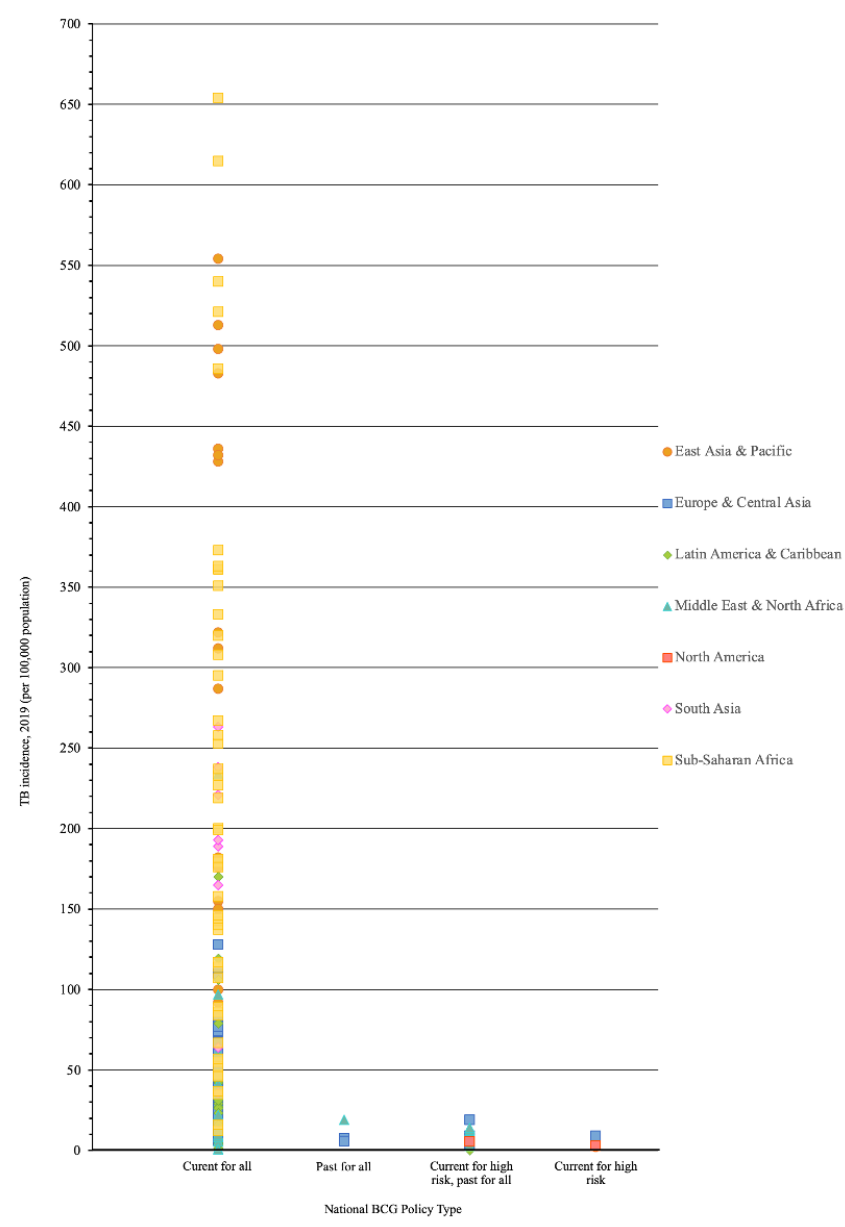

Figure 2 National BCG policy types for countries in the BCG Atlas by TB incidence rates (per 100000 population) in 2019 and WB region. TB cumulative incidence data (per 100 000) in 2019. Current national BCG policy types obtained from the BCG Atlas. TB, tuberculosis; WB, World Bank.

incidence rates were graphed before and after the policy change to examine any visual patterns.

The inter-rupted time series (ITS) design is increasingly being used to evaluate population-level health outcomes after a public health intervention, including legislation. ${ }^{17}$ The ITS model can assess changes in long-term TB incidence rates after policy change, such as a steepening of a pre-existing decline. To meet the quality criteria for ITS analysis, only countries with at least three data points before and after policy change were used. ${ }^{18}$

An ITS model was used to investigate whether changes to national BCG policies were associated with a decrease in yearly incidence rates. ${ }^{19}$ For the purposes of this analysis, it was assumed the policy change occurred at the beginning of the year and that it would take 1 year to implement at the national level; therefore, year of change was considered as 1 year prior to change.

\section{The BCG World Atlas .3.0}

The BCG Atlas now includes data for 194 different countries, with updated data in 2020 for 188 countries.
Countries are currently grouped into four BCG policy types: (1) current national vaccination for all neonates, (2) past national vaccination for all neonates with no high-risk groups identified, (3) current vaccination for high-risk groups and past vaccination for all neonates and (4) countries that only recommend vaccination for high-risk groups.

Over $80 \%$ of countries $(n=156 / 194,80.4 \%)$ continue to recommend mass BCG vaccination for all neonates; all countries in the sub-Saharan Africa, Latin American and East/South Asian regions follow these vaccination guidelines (figure 2). Using WHO TB burden definitions $(<40$ cases/100 000), 51/156 countries are low TB burden. ${ }^{1}$ All countries who currently recommend targeted vaccination for high-risk groups or who have ceased vaccination completely, have TB incidence rates clustered below 20 cases per 100000 persons (online supplemental figure 1).

\section{Changes to national BCG vaccine policies and TB incidence}

Thirteen countries changed their national BCG policy between 2009 and 2019, all from the European region, except for Barbados. Most of these countries $(\mathrm{n}=9 / 13,69.2 \%)$ changed their national policy from providing universal BCG vaccination to vaccinating high-risk groups only, while Ireland ceased BCG vaccination completely, due to shortage of supply. Other countries modified their definition of highrisk groups; Belgium added mandatory BCG vaccination for healthcare professionals, while both France and Sweden removed this group from their high-risk personnel in the same year they changed to a targeted programme. In 2018, almost 10 years after an initial policy change to a targeted approach, Norway changed their policy from vaccinating all healthcare personnel and students to only a select group. Similarly, Slovenia only began recommending mandatory BCG vaccination for neonates with families who have immigrated from countries with high TB incidence in 2015, after a decade of targeted vaccination beginning in 2005 . The specifics described in official policy documents are listed in online supplemental table 1 since the definitions are heterogeneous.

To visualise the change in $\mathrm{TB}$ incidence rates over time for countries who changed their national BCG policy, a 10-year period graph was created (figure 3) In most of these countries, there appears to be a general decreasing trend for TB incidence rates, making it difficult to assess whether the change in BCG policy had any additional effect on these rates.

Adequate data were available for all countries except for Sweden(2017), who did not have enough data points available postlegislation. Except for Norway, Malta and Barbados (whose rates were already exceptionally low), all other countries had a significant decline ranging between $0.13 \%$ and $1.35 \%$ in their national TB incidence rates before the policy change. In Norway, Czech Republic, France, Spain, Slovakia 


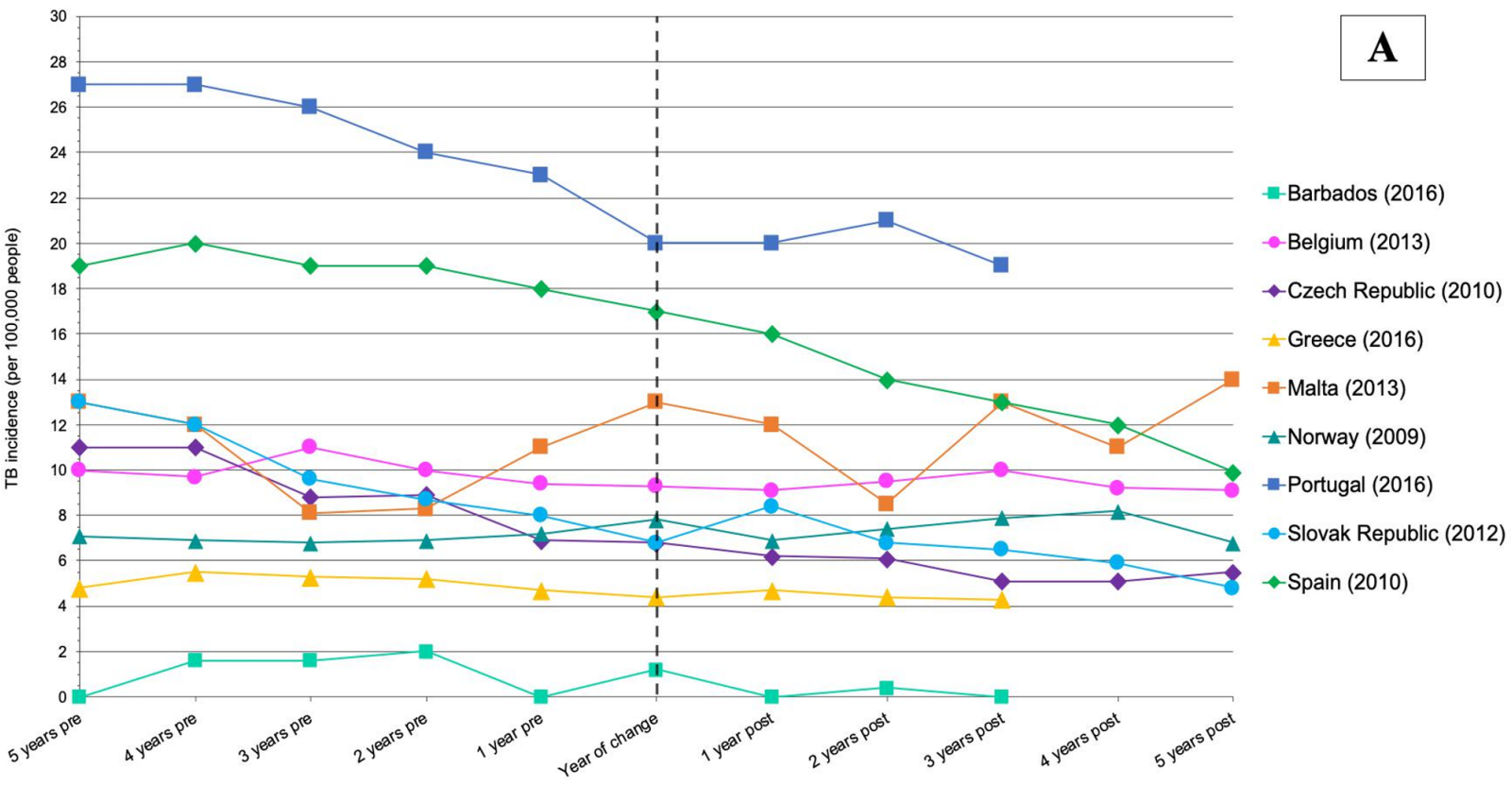

Years of Chanae

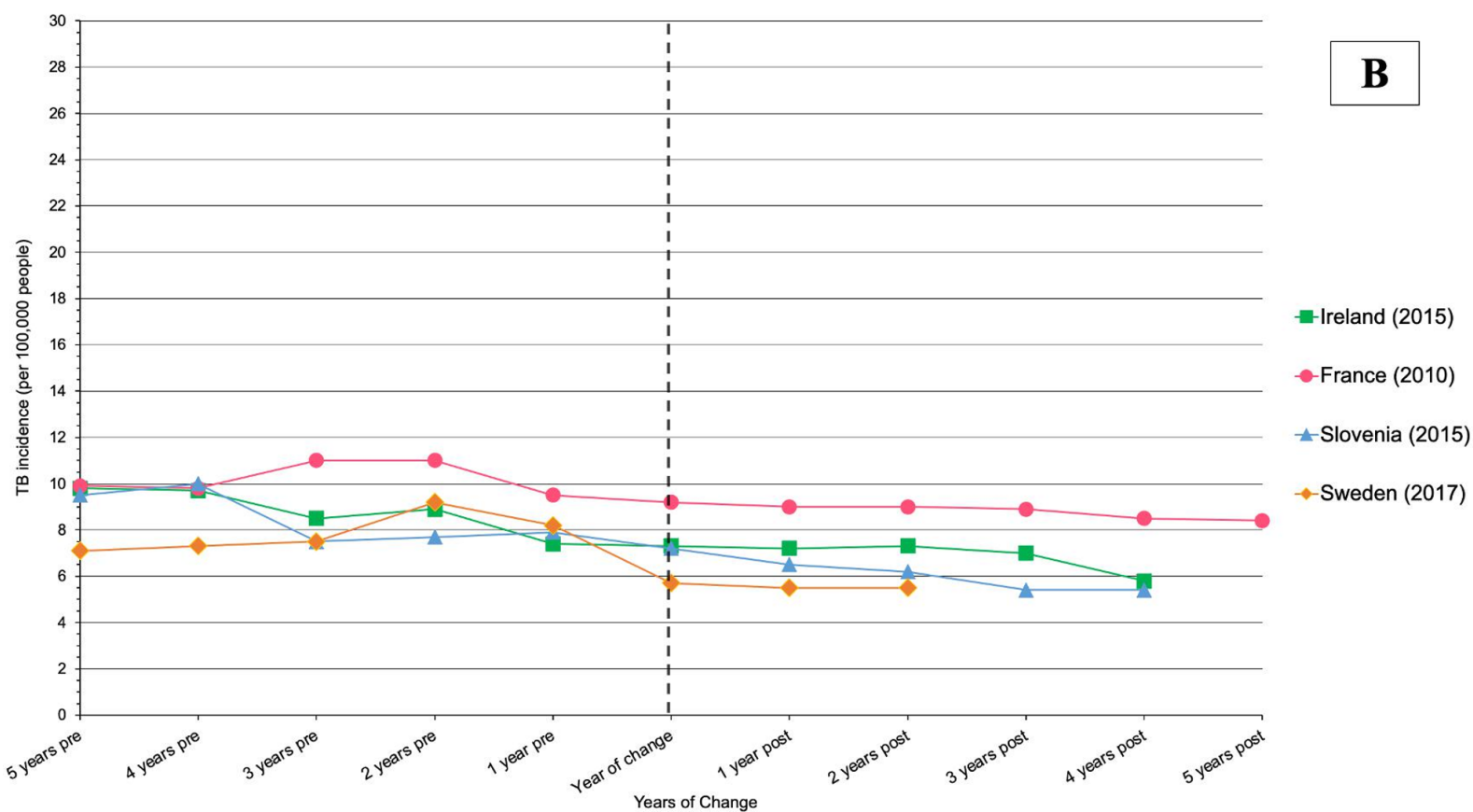

Figure 3 Countries who changed their national BCG policy type between 2009 and 2019, compared with TB incidence rates (per 100 000) over time, by years of change. (A) All countries who have changed their national policy from administering one dose to all neonates to only vaccinating members of high-risk groups. (B) All countries who have changed their national policy from administering one dose to all neonates to ceasing vaccination (Ireland) or have changed their definition of high-risk group (France and Slovenia, Belgium is excluded from B as it is already presented in A). TB, tuberculosis.

and Slovenia, there was a statistically significant decline in TB incidence when comparing the prelegislation and postlegislation period. During postlegislation period, most countries $(n=9 / 1275.0 \%)$ who had an initial decrease in TB incidence before the policy change continued to have a decrease.

When we expanded our search in the BCG Atlas to include all countries who changed their BCG guidelines from mass vaccination to a targeted approach, we identified 30 countries between 1975 and 2019 (online supplemental figure 2). Most changes occurred after 2005 and were in Europe. There were two countries with unknown year of change (from Europe and the Middle East, respectively).

\section{BCG vaccine booster doses}

Seven countries recommend at least one booster dose as part of their vaccination schedule; except for Sri Lanka, all countries are in Europe/Central Asia WB region (online supplemental table 2 ). There were 11 countries 


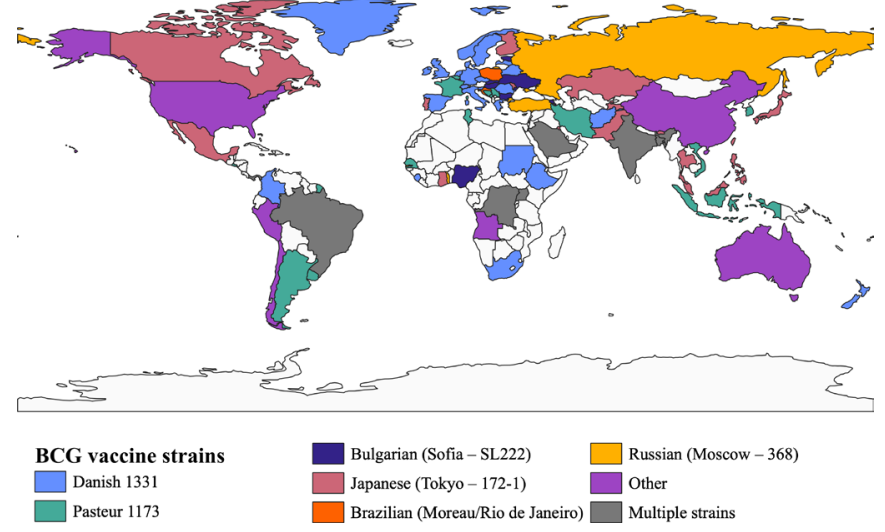

Figure 4 Global distribution of BCG vaccine strains for countries in the BCG Atlas in $2019(n=84)$. World map of BCG strains. Countries with no information about current strain usage are not highlighted.

who removed at least 1 booster dose from their guidelines between 2009 and 2019 (online supplemental table 2). Nine of these countries were in Europe and removed all of their booster doses from their guidelines $(n=9 / 11$, $81.8 \%$ ); Bulgaria and Russia removed their adolescent booster doses (14 and 17 years old, respectively), but kept those in childhood ( 7 and 12 years and 6 years old, respectively).

When we expanded our analysis to all countries that had removed booster doses, we identified 69 countries between 1970 and 2019, who were mostly in the European region $(n=53 / 69,76.8 \%)$ (online supplemental figure 3). Unfortunately, nine European countries did not identify the year of removal.

\section{BCG vaccine strains}

As shown in figure 4, we generated world maps to describe the global use and regional patterns of BCG vaccine strains. In the BCG Atlas, 84 countries had BCG strain information available. There are 10 different BCG substrains being used around the globe; Danish, Pasteur-1173, Bulgarian, Japanese, Brazilian, Russian, Connaught-Toronto, Mérieux, Chinese-Shanghai and TICE strain (figure 4). The Danish strain is the most common; there are 25 countries who listed it as their primary strain. Most of these countries are in the European region $(n=16 / 25,64.0 \%)$ followed by sub-Saharan Africa. Six countries (Bangladesh, DRC, India, Panama, Saudi Arabia, Uganda) are currently using multiple BCG strains. Unfortunately, BCG strain information remains unknown for most countries $(\mathrm{n}=107 / 191,56.0 \%)$.

\section{BCG vaccine stockouts}

BCG vaccine stockouts have occurred all over the globe for the past decade. Information regarding stockouts was available for 177 countries $(n=177 / 191,92.7 \%)$. Figure 5 illustrates global patterns of vaccine stockouts before (2009-2012), during (2013-2016) and after (2017-2019) the major stockout period. Between 2009 and 2012, there were approximately 30 countries every year who

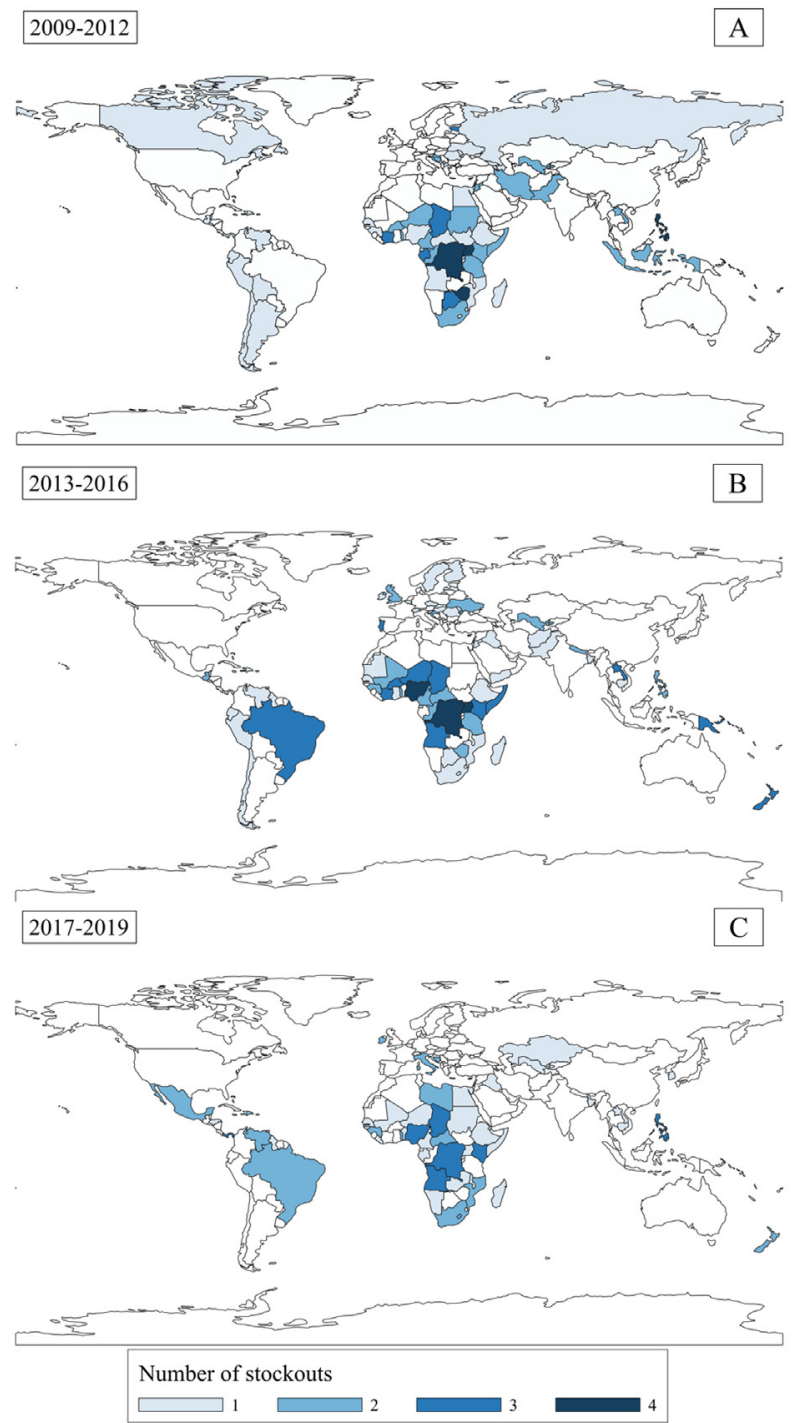

Figure 5 Global distribution of BCG vaccine stockouts for countries in the BCG atlas, by year (2009-2019). Countries who experienced at least one national or district BCG vaccine stockout: (A) between 2009 and 2012. (B) between 2013 and 2016. C) between 2017 and 2019.

experienced at least one stockout. The repercussions of the major stockout began in 2014 with the main effects felt in 2015; 56 countries reported stockouts, with over $40 \%$ of them never reporting a stockout prior to 2014 $(\mathrm{n}=25 / 56,44.6 \%)$. In 2016 , over $60 \%$ of countries who reported stockouts linked them to 2014-2015 ( $\mathrm{n}=17 / 27$, $62.9 \%$ ). During 2017-2019, the number of countries reporting at least one stockout dropped back to approximately 29 per year. Over the 10-year period, there were 23 countries who had reported at least five stockouts, all from sub-Saharan Africa, East Asia and Latin America.

\section{LESSONS LEARNT}

Our findings highlight the heterogeneity of BCG policies and practices worldwide. Over the last decade, Europe has had the fastest declining TB incidence rate among 
all WHO regions. ${ }^{20}{ }^{21}$ Barbados followed Trinidad and Tobago and the Bahamas, who also recorded no TB incident cases the year prior to policy change. ${ }^{22}$ Ireland ceased BCG vaccination, citing low incidence rates and low vaccine supply due to stockouts. ${ }^{6}$ Understanding the relationship and thresholds between TB incidence, stockouts and policy recommendations is an important association that should be looked at in future studies.

Our analysis also highlights the association between TB incidence and policy changes over time. The declining trend in TB incidence for most countries $(n=10 / 12$, $83.3 \%$ ) continued after the BCG policy change. This is an important finding as this strengthens the evidence about the effect and impact that BCG policies can have on incidence rates. This may quell uncertainty for low-burden countries about a possible large increase in TB incidence after changing from a universal programme to targeting high-risk groups. Future studies should investigate the impact of BCG policy changes not only at a national level but also regionally and in different subpopulations.

Although decreasing TB burden may be an indicator for switching to targeted BCG vaccination, it can present a new set of challenges. There is a potential risk for incomplete vaccination coverage among high-risk groups due to difficulties in identifying and defining the target population. ${ }^{6}$ This may justify low-incident countries in continuing mass vaccination. Changing to a targeted approach requires strong $\mathrm{TB}$ control programmes, adequate surveillance and identification of high-risk groups and resources to evaluate and implement changes to national policies and infrastructure. ${ }^{623}$ From a risk-benefit perspective, maintaining national vaccination has minimal risks and correlations between high vaccine coverage, reduced TB incidence and TB-associated mortality are well established. ${ }^{24}$ Historical examples of discontinuing mass vaccination in Czech Republic (2010) and Slovakia (2012), and subsequent increases in TB cases, may also explain hesitancy in changing vaccination policies. ${ }^{25}{ }^{26}$ Future research investigating changes in national BCG vaccine policy and practice, should also document the reasoning behind these changes to better understand their findings.

There are major disparities in incident $\mathrm{TB}$ rates and TB control programmes between Eastern and Western Europe. ${ }^{921}$ Countries who currently recommend booster doses were primarily in Eastern Europe and focused on school-aged children; school booster guidelines were historically created to ensure high vaccine coverage and thus may still be recommended due to weaknesses in national neonatal vaccination practices or other public health infrastructure. ${ }^{27}$

BCG strains have been considered interchangeable; however, recent evidence suggests they may confer different efficacy. ${ }^{28}$ A possible consequence of BCG stockouts may be a heterogeneous strain population; countries may change their BCG suppliers or have multiple suppliers to keep up with demand and thus, future populations would be immunised with a different strain. ${ }^{30}$ As the association between strain and vaccine efficacy is further investigated, this study highlights the importance of documenting BCG vaccine strain usage at the national and global level.

With only 2-4 suppliers providing over half the global supply of BCG, countries with universal vaccinations rely on these suppliers and are the most vulnerable for interruptions. ${ }^{31}$ Across Africa, $41 \%$ of countries reported a stockout every year since $2004 .^{10}$ Unfortunately, this region has the greatest TB incidence and risk for meningeal TB, for which BCG vaccine is protective. To avoid future catastrophic global shortages and prolonged suspension of vaccination, centralised information systems of vaccine supplies should be implemented as preventive measures and can aid in monitoring manufacturers to ensure the global demand is met. ${ }^{31-33}$

Given the limited effectiveness of BCG vaccine in adults, there is a clear and urgent need for the development of a new TB vaccine. ${ }^{34}$ As of August 2020, there are 14 vaccine candidates being evaluated at various clinical trials phases, but only 2 are in the final stage of testing. ${ }^{1}$ Some candidates will act as boosters in populations who have already received BCG vaccine underscoring the importance of the resources such as the BCG World Atlas. Promising candidates such as the M72/AS01E vaccine (GSK), demonstrating the potential to reduce TB disease by half in infected adults, should be prioritised and moved to the next phase..$^{35}$

\section{CONCLUSION}

We were able to assess the current global situation of BCG practices with the use of a unique database and data extraction methods (ie, data hackathon). We were also able to explore TB incidence trends over time and the potential impact of national policy changes. However, it is possible that countries which experienced a decrease in TB incidence could have been attributed to other TB control programmes, such as new screening and treatment methods. Further, as TB incidence data is collected annually, it may fail to show short-term changes and may mask varying incidence rates in different regions of the country.

Although the BCG Atlas is an extensive online database, there were several countries in which we had limited or no information. While every effort was made to improve completeness of the database prior to this analysis, some gaps remain and may influence the global BCG vaccination landscape.

BCG vaccination practices will continue to evolve along with global TB epidemiology. When evaluating policy changes, it is important to consider international best practices, evidence-based research and public health recommendations. Having more publicly available documents about policy changes can help future studies better understand the reasons behind them. This work has flagged several areas for more research and infrastructure strengthening that would benefit the global community. 
The BCG World Atlas continues to be a powerful tool for clinical, research and public health communities. By maintaining up to date information, this database can be used to track changes in practices over time. This may be helpful for TB policy-makers as they consider future changes to BCG vaccination guidelines, and as $\mathrm{TB}$ vaccine developers work towards a new and improved vaccine for $\mathrm{TB}$.

Twitter Samantha Lancione @samlancione, Hannah Alsdurf @hannahalsdurf and Madhukar Pai @paimadhu

Acknowledgements We would like to thank Dr Anita Dewaard, Dr Radoslav Kirkov and Mr. Adrian Wright for their work on the data hackathon, which contributed to the update of the BCG World Atlas. We would also like to thank Jeffrey Pennington, who provided computer support for the 3rd edition of the BCG World Atlas. We would like to thank all the contributors, respondents and volunteers who participated in the hackathon and directly through the BCG Atlas Website.

Contributors The study and its methodology were conceived by SL and AAZ. SL, JVA, AAZ performed the data collection, and SL and AAZ performed the analysis. All authors reviewed and provided feedback for the manuscript. AAZ is the study guarantor.

Funding The authors have not declared a specific grant for this research from any funding agency in the public, commercial or not-for-profit sectors.

Map disclaimer The inclusion of any map (including the depiction of any boundaries therein), or of any geographic or locational reference, does not imply the expression of any opinion whatsoever on the part of BMJ concerning the legal status of any country, territory, jurisdiction or area or of its authorities. Any such expression remains solely that of the relevant source and is not endorsed by BMJ. Maps are provided without any warranty of any kind, either express or implied.

Competing interests None declared.

Patient consent for publication Not applicable.

Ethics approval All tuberculosis incident and BCG vaccine data are publicly available and aggregate level with no identifying features. Therefore, research ethics board (REB) approval at the University of Ottawa to use this data was waived.

Provenance and peer review Not commissioned; externally peer reviewed.

Data availability statement Data are available in a public, open access repository: www.bcgatlas.org.

Supplemental material This content has been supplied by the author(s). It has not been vetted by BMJ Publishing Group Limited (BMJ) and may not have been peer-reviewed. Any opinions or recommendations discussed are solely those of the author(s) and are not endorsed by BMJ. BMJ disclaims all liability and responsibility arising from any reliance placed on the content. Where the content includes any translated material, BMJ does not warrant the accuracy and reliability of the translations (including but not limited to local regulations, clinical guidelines, terminology, drug names and drug dosages), and is not responsible for any error and/or omissions arising from translation and adaptation or otherwise.

Open access This is an open access article distributed in accordance with the Creative Commons Attribution Non Commercial (CC BY-NC 4.0) license, which permits others to distribute, remix, adapt, build upon this work non-commercially, and license their derivative works on different terms, provided the original work is properly cited, appropriate credit is given, any changes made indicated, and the use is non-commercial. See: http://creativecommons.org/licenses/by-nc/4.0/.

\section{ORCID iDs}

Samantha Lancione http://orcid.org/0000-0002-5081-5123

Hannah Alsdurf http://orcid.org/0000-0001-9801-7852

Madhukar Pai http://orcid.org/0000-0003-3667-4536

Alice Anne Zwerling http://orcid.org/0000-0003-1613-8525

\section{REFERENCES}

1 World Health Organization. WHO | Global tuberculosis report 2020, 2020. Available: http://www.who.int/tb/publications/global_report/ en/
2 Zwerling A, Behr MA, Verma A, et al. The BCG world atlas: a database of global BCG vaccination policies and practices. PLoS Med 2011;8:e1001012.

3 Colditz GA, Brewer TF, Berkey CS. Efficacy of BCG vaccine in the prevention of tuberculosis: meta-analysis of the published literature. Database of Abstracts of reviews of effects (DARE): quality-assessed reviews. York, UK: Centre for Reviews and Dissemination (UK), 1994. https://www.ncbi.nlm.nih.gov/books/NBK66270/

4 The BCG World Atlas. A database of global BCG vaccination policies and practices, 2020. Available: http://www.bcgatlas.org/index.php

5 et alPai M, Zwerling A, Badar S. Tracking TB vaccination policies and practices through space and time [Internet]. Nature portfolio microbiology community, 2017. Available: https://naturemicrobiolo gycommunity.nature.com/posts/15729-tracking-tb-vaccinationpolicies-and-practices-through-space-and-time

6 Faust L, Schreiber Y, Bocking N. A systematic review of BCG vaccination policies among high-risk groups in low TB-burden countries: implications for vaccination strategy in Canadian Indigenous communities. BMC Public Health 2019;19:1504.

7 Pooransingh S, Sakhamuri S. Need for BCG vaccination to prevent TB in high-incidence countries and populations. Emerg Infect Dis 2020;26:624-5

8 Arnesen TM, Brantsæter AB, Nøkleby H. Bacille Calmette-Guérin vaccination of healthcare personnel: changing epidemiology calls for new recommendations in Norway. Eur Respir J 2019;53:1801123.

9 European Respiratory Society. Bacille Calmette-Guérin vaccination: the current situation in Europe, 2021. Available: https://erj. ersjournals.com/content/43/1/24.long

10 Cernuschi T, Malvolti S, Nickels E, et al. Bacillus Calmette-Guérin (BCG) vaccine: a global assessment of demand and supply balance. Vaccine 2018;36:498-506.

11 Zwerling A, Lancione S. The BCG Atlas: Using data science to improve knowledge around a century old vaccine [Internet]. Nature Portfolio Microbiology Community, 2020. Available: http://naturemi crobiologycommunity.nature.com/posts/the-bcg-atlas-using-datascience-to-improve-knowledge-around-a-century-old-vaccine

12 Elsevier. BCG-COVID 19 Hackathon: task 1 winners announced, task 2 - data scientists - we need you! 2021. Available: https:// pharma.elsevier.com/covid-19/bcg-covid-19-hackathon-task-1winners-announced-task-2-data-scientists-we-need-you/

13 STAT. Why an old TB vaccine is getting attention in the fight against Covid-19, 2020. Available: https://www.statnews.com/2020/04/14/ decades-old-tb-vaccine-attracts-attention-and-skepticism-as-apotential-weapon-against-covid-19/

14 Weiss S. Does the BCG vaccine work against coronavirus? We just don't know. Wired UK, 2020. Available: https://www.wired.co.uk/ article/bcg-vaccine-coronavirus-uk

15 UMC Utrecht. Tuberculosis vaccine does not protect vulnerable elderly people against COVID-19, 2021. Available: https://www. umcutrecht.nl/en/over-ons/nieuws/algemeen/tuberculosisvaccine-does-not-protect-vulnerable-elderly-people-againstcovid-19

16 Chimoyi L, Velen K, Churchyard GJ, et al. An ecological study to evaluate the association of Bacillus Calmette-Guerin (BCG) vaccination on cases of SARS-CoV2 infection and mortality from COVID-19. PLoS One 2020;15:e0243707.

17 Kontopantelis E, Doran T, Springate DA, et al. Regression based quasi-experimental approach when randomisation is not an option: interrupted time series analysis. BMJ 2015;350:h2750.

18 The Cochrane Collaboration- EPOC group. Including interrupted time series (ITS) designs in a EPOC review, 1998. Available: https:// epoc.cochrane.org/sites/epoc.cochrane.org/files/public/uploads/ inttime.pdf

19 Bernal JL, Cummins S, Gasparrini A. Interrupted time series regression for the evaluation of public health interventions: a tutorial. Int J Epidemiol 2017;46:348-55.

20 Walls T, Shingadia D. The epidemiology of tuberculosis in Europe. Arch Dis Child 2007;92:726-9.

21 Infuso A, Falzon D. EuroTB network. European survey of BCG vaccination policies and surveillance in children, 2005. Euro Surveill Bull Eur Sur Mal Transm Eur Commun Dis Bull 2006;11:6-11.

22 Altink H. 'Fight TB with BCG': mass vaccination campaigns in the British Caribbean, 1951-6. Med Hist 2014;58:475-97.

23 Feiring B, Laake I, Molden T, et al. Do selective immunisation against tuberculosis and hepatitis $\mathrm{B}$ reach the targeted populations? A nationwide register-based study evaluating the recommendations in the Norwegian childhood immunisation programme. Vaccine 2016;34:2015-20.

24 Saad-Hussein A, Mohammed AM. Trend of application of World Health organization control strategy of tuberculosis in Egypt. $J$ Epidemiol Glob Health 2014;4:195-202. 
25 Samohyl M, Solovic I, Svecova J, et al. Tuberculosis as public health problem in the Slovak Republic. Iran J Public Health 2017;46:852-3.

26 World Health Organization. Combating tuberculosis in Slovakia is a battle not yet won, 2014. Available: https://www.euro.who.int/en/ countries/slovakia/news/news/2014/04/combating-tuberculosis-inslovakia-is-a-battle-not-yet-won

27 Mangtani P, Nguipdop-Djomo P, Keogh RH, et al. The duration of protection of school-aged BCG vaccination in England: a population-based case-control study. Int J Epidemiol 2018;47:193-201.

28 Shann F. Substantial benefits from finding the most effective BCG strain. Lancet Respir Med 2016;4:e35.

29 Abdallah AM, Hill-Cawthorne GA, Otto TD, et al. Genomic expression Catalogue of a global collection of BCG vaccine strains show evidence for highly diverged metabolic and cell-wall adaptations. Sci Rep 2015;5:15443.
30 Ritz N, Curtis N. Mapping the global use of different BCG vaccine strains. Tuberculosis 2009;89:248-51.

31 Harris RC, Dodd PJ, White RG. The potential impact of BCG vaccine supply shortages on global paediatric tuberculosis mortality. BMC Med 2016;14:138.

32 Bateman C. Vaccines: SA's immunisation programme debunked. S Afr Med J 2016;106:318-9.

33 Gavilanes AM. Vaccines that don't arrive [Internet]. Medicamentalia, 2017. Available: https://medicamentalia.org/vaccines/stockout/

34 Faust L, Raithby L. The 100th anniversary of a vaccine against a deadly disease: not a cause for celebration [Internet]. Health Affairs Blog, 2021. Available: https://www.healthaffairs.org/do/10.1377/ hblog20210503.703348/full/

35 Tait DR, Hatherill M, Van Der Meeren O, et al. Final Analysis of a Trial of M72/AS01 Vaccin to Prevent Tuberculosis. N Engl J Med 2019;381:2429-39. 